\title{
Ellagic acid induces HeLa cell apoptosis via regulating signal transducer and activator of transcription 3 signaling
}

\author{
LIAN-WEI LI ${ }^{1}$, CHAO NA $^{2}$, SONG-YU TIAN ${ }^{1}$, JIE CHEN $^{1}$, RONG MA $^{1}$, YING GAO $^{1}$ and GE LOU ${ }^{1}$ \\ ${ }^{1}$ Department of Gynecology, Harbin Medical University Cancer Hospital, Harbin, Heilongjiang $150081 ;{ }^{2}$ Department of \\ Integrated TCM and Western Medicine, Heilongjiang Provincial Hospital, Harbin, Heilongjiang 150036, P.R. China
}

Received October 13, 2016; Accepted June 6, 2017

DOI: $10.3892 /$ etm.2018.6182

\begin{abstract}
Ellagic acid has been reported to possess various activities, including anti-inflammatory, anti-oxidative, antiviral and anticancer abilities. However, the effect and underlying molecular mechanism of ellagic acid on cervical carcinoma remain unclear. Therefore, the present study aimed to investigate the effects of ellagic acid on human cervical carcinoma cells and the molecular mechanism involved. The present study assessed the survival of HeLa cells cultured in vitro using an MTT assay. Apoptosis rate and cell cycle of HaLa cells were measured using an Annexin V-Fluorescein isothiocyanate/propidium iodide Apoptosis Detection and Cell Cycle Analysis kits, respectively, following treatment with varying concentrations of ellagic acid. Further effects of ellagic acid on HeLa cells was assessed using flow cytometry and western blotting. Ellagic acid treatment significantly inhibited cell proliferation of the human cervical carcinoma HeLa, SiHa and C33A cells. In HeLa cells, it was observed that ellagic acid arrested the cell cycle at G1 phase, induced cell apoptosis, suppressed the phosphorylation of Janus kinase 2 and signal transducer and activator of transcription 3 (STAT3), as well as modulated the expression of associated proteins. Collectively, the results of the present study provide evidence that ellagic acid inhibits cervical carcinoma cell proliferation, and induces apoptosis and cell cycle arrest at G1 phase possibly via the regulation of STAT3 signaling.
\end{abstract}

\section{Introduction}

Cervical carcinoma represents the fourth most common cancer type worldwide in women, and the third highest cause

Correspondence to: Dr Ge Lou, Department of Gynecology, Harbin Medical University Cancer Hospital, 150 Haping Road, Nan Gang, Harbin, Heilongjiang 150081, P.R. China

E-mail: gelou4526@sina.com

Key words: ellagic acid, cervical carcinoma, signal transducer and activator of transcription 3, HeLa cells, apoptosis of cancer-associated mortality among females in developing countries (1). According to statistical data, there were 527,600 new cervical carcinoma cases and 265,700 associated mortalities worldwide in 2012 (2). Current therapeutic strategies of cervical carcinoma, including surgery, radiation therapy and chemotherapy, have a high recurrence rate, limited effectiveness and various side effects. Thus, it is urgent to explore novel potential therapies for enhancing the prevention and treatment of cervical carcinoma.

Recently, numerous scholars focused on exploring natural compounds for the treatment of cervical cancer, due to their multiple pharmacological activities and low side effects (3-5). Ellagic acid, with the chemical formula 4,4',5,5',6,6'-hexahydroxydiphenic acid-2,6,2',6'-dilactone, is widely found in various fruits and plants, including pomegranates, strawberries, raspberries and blackberries (6). The structure of ellagic acid is shown in Fig. 1A. Ellagic acid has been reported to possess various effects, including anti-inflammatory (7), anti-oxidative (8) and antiviral (9) activities. Previous studies have demonstrated that ellagic acid was able to inhibit tumor growth in a human pancreatic cancer cell xenografted mice (10), and to induce cell apoptosis in TSGH8301 human bladder cancer cells via endoplasmic reticulum stress- and mitochondria-dependent signaling pathways (11). In addition, ellagic acid potentiated the differentiation of human leukemia cells (12) and presented an anti-angiogenesis activity in breast cancer (13), suggesting the strong anticancer activity of ellagic acid. Furthermore, a previous study indicated that ellagic acid treatment inhibited cell growth and induced cell apoptosis in human cervical carcinoma CaSki cells (14). However, the molecular mechanism underlying the effect of ellagic acid on cervical carcinoma remains unclear. A recent study demonstrated that ellagic acid significantly decreased cell proliferation via its inhibitory effects on signal transducer and activator of transcription 3 (STAT3) signaling in human prostate cancer cells (15). Additionally, it is widely identified that STAT3, which functions as an oncogenic transcription factor, serves a key role in regulating tumor cell proliferation, survival, apoptosis and angiogenesis in numerous types of cancer $(16,17)$. Clinical and pro-clinical studies have noted that STAT3 is aberrantly expressed in cervical carcinoma tissues and cell lines $(18,19)$. Increasing evidence also demonstrated that STAT3 serves a key role in the tumor microenvironment (20) and human papillomaviruses 
infection $(18,21)$ during the development of cervical cancer. Based on the aforementioned observations, it is hypothesized that ellagic acid may inhibit human cervical carcinoma cells, and the mechanism may be associated with the regulation of STAT3 signaling.

Therefore, the current study aimed to investigate the effect and molecular mechanism of ellagic acid on cervical carcinoma cells. Herein, it was observed that ellagic acid significantly inhibited the growth of human cervical carcinoma cell lines, induced cell apoptosis and arrested the cell cycle at G1 phase in human HeLa cells, possibly by regulating the activation of STAT3 signaling.

\section{Materials and methods}

Chemicals and antibodies. Dulbecco's modified Eagle's medium (DMEM), fetal bovine serum (FBS), penicillin and streptomycin were purchased from Thermo Fisher Scientific, Inc. (Waltham, MA, USA). Ellagic acid, 3-(4,5-Dimethylthiazol2-yl)-2,5-diphenyltetrazolium bromide (MTT) and propidium iodide (PI) were purchased from Sigma-Aldrich (Merck KGaA, Darmstadt, Germany). Antibodies against phosphorylated-Janus kinase 2 (p-JAK2; cat. no. 3771), p-STAT3 (Ser727; cat. no. 94994), p-STAT3 (Tyr705; cat. no. 9145), CyclinD1 (cat. no. 2922), B-cell lymphoma-extra large (Bcl-xl; cat. no. 2764) and myeloid cell leukemia 1 (Mcl-1; cat. no. 5453) were obtained from Cell Signaling Technology, Inc. (Danvers, MA, USA; All 1:1,000). Since Tyr705 is required for STAT3 activation and the present study involves the Ser727 signaling pathway, Ser727 and Tyr705 (two sites of STAT3) were examined. Antibodies against glyceraldehyde-3-phosphate dehydrogenase (GAPDH; cat. no. sc-365062) and horseradish peroxidase (HRP)-conjugated anti-rabbit IgG (cat. no. sc-2005) were purchased from Santa Cruz Biotechnology, Inc. (Santa Cruz, CA, USA; all 1:5,000). All other chemicals and reagents were of analytical grade.

Cell culture. Human cervical carcinoma HeLa, SiHa and C33A cells were obtained from the Shanghai Cell Bank (Shanghai, China). HeLa, SiHa and C33A cells were cultured in DMEM supplemented with $10 \% \mathrm{FBS}, 100 \mathrm{U} / \mathrm{ml}$ penicillin and $100 \mu \mathrm{g} / \mathrm{ml}$ streptomycin at $37^{\circ} \mathrm{C}$ under a humidified $5 \%$ $\mathrm{CO}_{2}$ atmosphere.

Cell viability assay. MTT assay was performed to investigate the cell proliferation. Briefly, cells were seeded in 96-well plates at a density of $2 \times 10^{3}$ cells per well and cultured overnight at $37^{\circ} \mathrm{C}$. The control group was treated with $0.1 \%(\mathrm{v} / \mathrm{v})$ dimethyl sulfoxide (DMSO), serving as the vehicle. The cells were then incubated at $37^{\circ} \mathrm{C}$ with different concentrations $(0$, $10,20$ and $30 \mu \mathrm{M})$ of ellagic acid at different time points (24, 48 and 72 h). Subsequently, $20 \mu \mathrm{l} \mathrm{MTT}(5 \mathrm{mg} / \mathrm{ml})$ were added to each well and incubated for an additional $3 \mathrm{~h}$ at $37^{\circ} \mathrm{C}$. Following lysis with $150 \mu 1 \mathrm{DMSO}$, the absorbance was detected using a microplate reader (Thermo Fisher Scientific, Inc.).

Cell apoptosis detection. HeLa cells were treated with different concentrations of ellagic acid for $72 \mathrm{~h}$. Next, cell apoptosis was evaluated with an Annexin V-FITC/PI Apoptosis Detection kit (BD Pharmingen, San Diego, CA, USA) according to the manufacturer's instructions. The apoptosis ratio was then measured using a Beckman Coulter CyAn ADP Flow Cytometer (Becton Dickinson, San Jose, CA, USA).

Cell cycle progression examination. HeLa cells were treated with different concentrations $(0,10,20$ and $30 \mathrm{uM})$ of ellagic acid for $72 \mathrm{~h}$ and cell apoptosis was evaluated with a Cell Cycle Analysis kit (BD Pharmingen) following the instructions provided by the manufacturer. Briefly, subsequent to treating with various concentrations of ellagic acid for $48 \mathrm{~h}$, HeLa cells were trypsinized and centrifuged at a speed of $1,000 \mathrm{x} \mathrm{g}$ for $10 \mathrm{~min}$ at room temperature. The pellet was washed three times with phosphate-buffered saline and fixed with $70 \%$ alcohol. Subsequently, the nuclei were stained with PI and the DNA content was measured by flow cytometry (Becton Dickinson) in accordance with a previous study (22).

Western blot analysis. Following incubation with various concentrations of ellagic acid for $72 \mathrm{~h}$, the total protein was extracted from HeLa cells and the protein concentrations were determined using a is Pierce ${ }^{\mathrm{TM}}$ BCA Protein assay kit (cat. no. 23225; Bio-Rad Laboratories, Inc., Hercules, CA, USA). Equal amounts of protein $(20 \mu \mathrm{g})$ were separated by $10 \%$ sodium dodecyl sulfate-polyacrylamide gel electrophoresis and then transferred to polyvinylidene difluoride membranes. Blocking was then performed by overnight incubation in Tris-buffered saline/Tween 20 (TBST) containing 5\% non-fat dried milk. The membranes were then incubated with the primary antibodies against p-JAK2, p-STAT3 (Ser727), p-STAT3 (Tyr705), CyclinD1, Bcl-xl, Mcl-1 and GAPDH overnight at $4^{\circ} \mathrm{C}$. Subsequent to washing with TBST, the blots were incubated with the aforementioned secondary antibodies coupled to HRP for $1 \mathrm{~h}$ at $37^{\circ} \mathrm{C}$. The signal was visualized with an enhanced chemiluminescence kit (EMD Millipore, Billerica, MA, USA) and GAPDH was used as an internal loading control. The gray value of the target protein and GAPDH were analyzed using Image $\mathbf{J}$ software (version 4.0; National Institutes of Health, Bethesda, MD, USA).

Statistical analysis. The data are expressed as the mean \pm standard deviation. All experiments were performed three times and the data were analyzed by the GraphPad Prism 5 (GraphPad Software, Inc., La Jolla, CA, USA). Statistical analysis between different groups was performed using the one-way analysis of variance (ANOVA) followed by a post-hoc Bonferroni test. A two-way ANOVA was used in the cell proliferation (MTT) assay. $\mathrm{P}<0.05$ was considered to indicate a statistically significant difference.

\section{Results}

Effect of ellagic acid on cell proliferation of human cervical carcinoma cells. To investigate the effect of ellagic acid on cell proliferation of human cervical carcinoma cells, HeLa, $\mathrm{SiHa}$ and $\mathrm{C} 33 \mathrm{~A}$ cells were treated with various concentrations of ellagic acid for 24,48 or $72 \mathrm{~h}$. As ellagic acid treatment time and concentration increased, the cell viability of the three cervical carcinoma cell lines, including HeLa (Fig. 1B), SiHa (Fig. 1C) and C33A cells (Fig. 1D) significantly decreased. 
A<smiles>O=c1oc2c(O)c(O)cc3c(=O)oc4c(O)c(O)cc1c4c23</smiles>

C

SiHa cells



B



D



Figure 1. Ellagic acid inhibits the cell proliferation of human cervical carcinoma cell lines. (A) Chemical structure of ellagic acid. Effects of ellagic acid on the growth of (B) HeLa, (C) SiHa and (D) C33A cells are shown. All data are expressed as the mean \pm standard deviation. ${ }^{\#} \mathrm{P}<0.05$, ${ }^{*} \mathrm{P}<0.05$ vs. control.

Ellagic acid stimulates cell apoptosis in HeLa cells. To evaluate the effect of ellagic acid on cell apoptosis of cervical carcinoma cells, HeLa cells were treated with various concentrations of ellagic acid and examined by flow cytometry. As illustrated in Fig. 2, ellagic acid dosage-dependently increased the number of apoptotic HeLa cells. In comparison with the NC group $(4.90 \pm 0.98 \%)$, the apoptosis rates upon treatment with 10 (16.3 $\pm 1.38 \%), 20(22.0 \pm 3.24 \%)$ and $30(32.1 \pm 2.27 \%) \mu \mathrm{M}$ ellagic acid were significantly increased $(\mathrm{P}<0.05)$. These observations suggest that ellagic acid induces cell apoptosis, which may contribute to the anticancer effect of ellagic acid in HeLa cells.

Effect of ellagic acid on cell cycle distribution of HeLa cells. The present study subsequently investigated whether ellagic acid was able to affect the cell cycle distribution of HeLa cells by flow cytometry with PI staining, and the results are shown in Fig. 3 and Table I. Ellagic acid at the three investigated doses induced G1 cell cycle arrest in HeLa cells, as evidenced by the significantly increased proportion of cells at the G1 phase $(69.9 \pm 3.55,75.7 \pm 3.27$ and $84.7 \pm 3.01 \%$ for 10,20 and $30 \mu \mathrm{M}$ ellagic acid, respectively; all $\mathrm{P}<0.05)$ in HeLa cells compared with the control group $(60.3 \pm 4.21 \%)$. The results demonstrated that the proportion of cells at the $\mathrm{G} 2$ phase were significantly decreased in HeLa cells compared with the control group $(20.4 \pm 2.94,15.6 \pm 2.87$ and $9.38 \pm 1.37$ for 10,20 and $30 \mu \mathrm{M}$ ellagic acid, respectively; all $\mathrm{P}<0.05)$.
Effect of ellagic acid on STAT3 signaling in HeLa cells. Given that STAT3 signaling serves a pivotal role in tumor cell proliferation, survival and apoptosis, the current study assessed the effect of ellagic acid on the expression levels of STAT3 and associated proteins by western blot analysis. As shown in Fig. 4, compared with the control. the cells treated with ellagic acid presented significant decreased levels ofp-JAK2 $(10 \mu \mathrm{M}, 0.59 \pm 0.066 ; 20 \mu \mathrm{M}, 0.44 \pm 0.089$; $30 \mu \mathrm{M}, 0.27 \pm 0.088)$, p-STAT3 (Ser727) (10 $\mu \mathrm{M}, 0.69 \pm 0.085$; $20 \mu \mathrm{M}, 0.49 \pm 0.097 ; 30 \mu \mathrm{M}, 0.39 \pm 0.086)$ and $\mathrm{p}-\mathrm{STAT} 3$ (Tyr705) $(10 \mu \mathrm{M}, 0.77 \pm 0.092 ; 20 \mu \mathrm{M}, 0.58 \pm 0.104$; and $30 \mu \mathrm{M}, 0.33 \pm 0.116)$, when compared with the control group levels $(1.00 \pm 0.186,1.00 \pm 0.129$ and $1.00 \pm 0.141$, respectively; all $\mathrm{P}<0.05)$. This indicates that ellagic acid treatment may suppress the activation of STAT3 signaling in HeLa cells. Furthermore, the protein expression levels of downstream genes were evidently downregulated by the administration of ellagic acid, including the levels of CyclinD1 $(10 \mu \mathrm{M}$, $0.81 \pm 0.107 ; 20 \mu \mathrm{M}, 0.55 \pm 0.073 ; 30 \mu \mathrm{M}, 0.34 \pm 0.073), \mathrm{Bcl}-\mathrm{xl}$ (10 $\mu \mathrm{M}, 0.73 \pm 0.060 ; 20 \mu \mathrm{M}, 0.58 \pm 0.049 ; 30 \mu \mathrm{M}, 0.31 \pm 0.079)$ and Mcl-1 $(10 \mu \mathrm{M}, 0.76 \pm 0.057 ; 20 \mu \mathrm{M}, 0.54 \pm 0.070$; and $30 \mu \mathrm{M}, 0.31 \pm 0.076)$, compared with the control group levels $(1.00 \pm 0.107,1.00 \pm 0.155$ and $1.00 \pm 0.156$; all $\mathrm{P}<0.05)$. These results suggest that ellagic acid induces cell cycle arrest at the G1 phase and that cell apoptosis may be induced via the suppression of STAT3 signaling. 



AnnexinV-FITC



Figure 2. Ellagic acid induces the apoptosis of HeLa cells. (A) Flow cytometry analysis of apoptosis in HeLa cells treated for $72 \mathrm{~h}$ with the indicated concentrations of ellagic acid. (B) Total percentage of apoptosis in HeLa cells in the different treatment groups. All data are expressed as the mean \pm standard deviation. ${ }^{*} \mathrm{P}<0.05$ vs. the control group. PI, propidium iodide.

\section{Discussion}

The current study provided insights into the effect of ellagic acid on the cell proliferation, cell cycle progression and apoptosis of HeLa cervical carcinoma cells. The most notable findings of the present study were that ellagic acid arrested the cell cycle at the G1 phase, induced cell apoptosis, suppressed the phosphorylationof JAK2 and STAT3, and modulated the expression of associated proteins. To the best of our knowledge, the current study is the first to demonstrate that ellagic acid exerts an anticancer activity in cervical carcinoma HeLa cells possibly by inducing cell apoptosis and arresting the cell cycle via the regulation of STAT3 signaling.

Ellagic acid, a plant polyphenolic compound, has been demonstrated to exert an antitumor activity in several types of cancer (23-25). The MTT assay conducted in the present study further confirmed the anticancer property of ellagic acid in human cervical carcinoma cell lines. It was demonstrated 

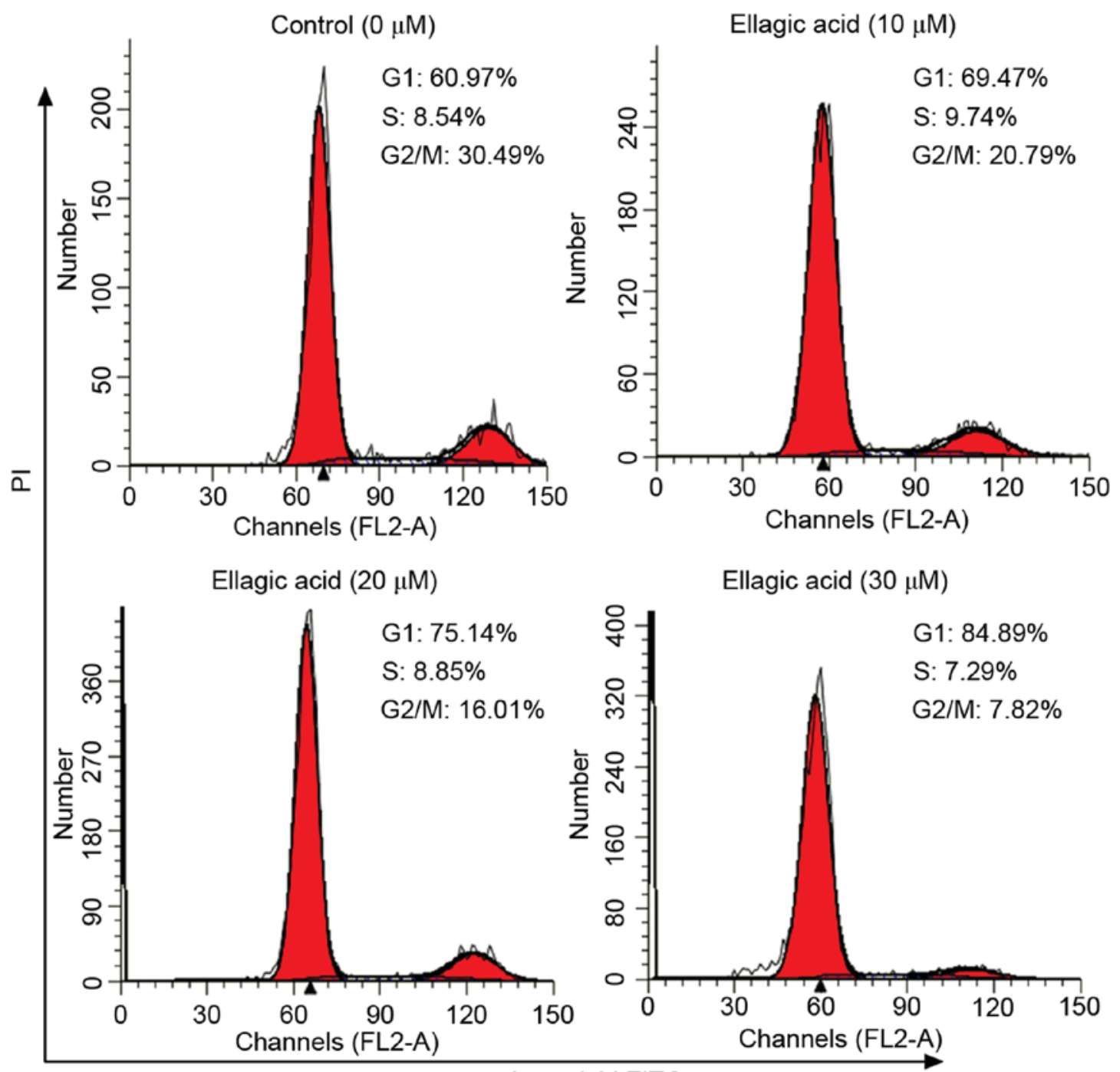

AnnexinV-FITC

Figure 3. Ellagic acid arrests the cell cycle in the G1 phase in HeLa cells, as observed by flow cytometry analysis of the different treatment groups. PI, propidium iodide.

that ellagic acid reduced the proliferation of the human cervical carcinoma HeLa, SiHa and C33A cell lines in a dosage- and time-dependent manner. Another study has previously reported that ellagic acid dose-dependently suppressed HeLa cell growth (26), which is in agreement with the current observations. Of note, the present study results revealed that the inhibitory effects on HeLa cells were more significant compared with those on $\mathrm{SiHa}$ and C33A cells, thus the HeLa cell line was used for subsequent experiments to explore the molecular mechanism underlying the effects of ellagic acid.

The mechanism of the anticancer effect of ellagic acid is widely considered to involve the regulation of the cell cycle and apoptosis. For instance, ellagic acid inhibited the growth of MCF-7 breast cancer cells via inducing G0/G1 arrest (27), as well as suppressed cell growth by arresting the cell cycle in the G1 phase and inducing cell apoptosis in ovarian carcinoma cells (28). To further examine the exact mechanism of ellagic acid-induced inhibition of HeLa proliferation, the effects of ellagic acid on HeLa cell cycle and apoptosis were determined by flow cytometry. According to these experiments, the present study observed that ellagic acid treatment markedly arrested the cell cycle in the G1 phase and stimulated cell apoptosis in HeLa cells at a dosage-dependent manner. Furthermore, STAT3 is generally identified as an oncogenic transcription factor, which is involved in the regulation of tumor cell proliferation, survival, apoptosis and angiogenesis in various types of cancer $(16,17)$. In the current study, it was revealed that the phosphorylation of JAK2 and STAT3 were significantly inhibited by treatment with ellagic acid, implying the inhibitory effect of ellagic acid on STAT3 signaling activation. Furthermore, STAT3 has been demonstrated to regulate tumorigenic proteins, including CyclinD1, Bcl-xl and Mcl-1 (29,30). CyclinD1, the major regulator of cyclin-dependent kinase (CDK) 4 or CDK6 activation, drives the progression from $\mathrm{G} 1$ to $\mathrm{S}$ phase (31). Notably, the results of the current study demonstrated that ellagic acid evidently downregulated the expression of CyclinD1, which is consistent with the results of the cell cycle assay. Bcl-xl and Mcl-1, two anti-apoptotic proteins involved in the apoptosis and survival of various cancer types, were also significantly decreased by the treatment with ellagic acid in HeLa cells. Collectively, the aforementioned observations indicate that ellagic acid induces 
Table I. Proportion of HeLa cells in G1, S and G2 phases of the cell cycle following ellagic acid treatment.

\begin{tabular}{lllcc}
\hline & & \multicolumn{3}{c}{ Ellagic acid dose $\mu \mathrm{M}$} \\
\cline { 3 - 5 } Cell cycle phase & Control & 10 & 20 & 30 \\
\hline G1 & $60.3 \pm 4.21$ & $69.9 \pm 3.55^{\mathrm{a}}$ & $75.7 \pm 3.27^{\mathrm{a}}$ & $84.7 \pm 3.01^{\mathrm{a}}$ \\
S & $8.44 \pm 0.39$ & $9.65 \pm 0.61$ & $8.71 \pm 0.41$ & $7.56 \pm 0.37$ \\
G2 & $31.2 \pm 3.82$ & $20.4 \pm 2.94^{\mathrm{a}}$ & $15.6 \pm 2.87^{\mathrm{a}}$ & $9.38 \pm 1.37^{\mathrm{a}}$ \\
\hline
\end{tabular}

${ }^{a} \mathrm{P}<0.05$ vs. the control group. All data are expressed as the mean \pm standard deviation.

A



D



$\mathrm{F}$



B



C



E

CyclinD1



G

Mcl-1

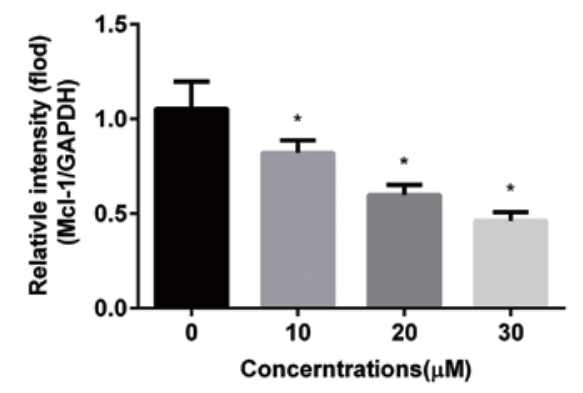

Figure 4. Ellagic acid suppresses STAT3 signaling pathway in HeLa cells. (A) Representative western blot bands of the proteins associated with STAT3 signaling. The protein expression levels of (B) p-JAK2, (C) p-STAT3 (Ser727), (D) p-STAT3 (Tyr705), (E) CyclinD1, (F) Bcl-xl and (G) Mcl-1 were detected by western blot analysis and normalized to GAPDH. All data are expressed as the mean \pm standard deviation.*P<0.05 vs. the control group. STAT3, signal transducer and activator of transcription 3; JAK2, Janus kinase 2; p-, phosphorylated; Bcl-xl, B-cell lymphoma-extra large; Mcl-1, myeloid cell leukemia 1; GAPDH, glyceraldehyde-3-phosphate dehydrogenase. 
cell cycles arrest in the G1 phase and cell apoptosis possibly via the suppression of STAT3 signaling. However, further experimental studies are required to validate these findings in vivo and to clarify the precise mechanism by which ellagic acid regulates STAT3 signaling in cervical carcinoma cells.

In conclusion, the data of the present study indicated that treatment with ellagic acid markedly inhibited cell proliferation, and induced G1 arrest and apoptosis by suppressing the activation of STAT3 signaling in cervical carcinoma HeLa cells. Thus, these findings suggest the potential application of ellagic acid for the management of patients with cervical carcinoma.

\section{Acknowledgements}

Not applicable.

\section{Funding}

The present study was supported by the Scientific Research Project of Hei Long-jiang Provincial Health Bureau of China (grant no. 2016-105) and the Administration of Traditional Chinese Medicine of Heilongjiang Province, China (grant no. ZHY16-110).

\section{Availability of data and materials}

The datasets used and/or analyzed during the current study are available from the corresponding author on reasonable request.

\section{Authors' contributions}

LL wrote the manuscript and analyzed the data; CN and ST analyzed the data and revised the manuscript; JC analyzed the data; RM and YG collected the data; and GL designed the study.

\section{Ethics approval and consent to participate}

Not applicable.

\section{Consent for publication}

Not applicable.

\section{Competing interests}

The authors declare that they have no competing interests.

\section{References}

1. Ferlay J, Soerjomataram I, Dikshit R, Eser S, Mathers C, Rebelo M, Parkin DM, Forman D and Bray F: Cancer incidence and mortality worldwide: Sources, methods and major patterns in GLOBOCAN 2012. Int J Cancer 136: E359-E386, 2015.

2. Torre LA, Bray F, Siegel RL, Ferlay J, Lortet-Tieulent J and Jemal A: Global cancer statistics, 2012. CA: a cancer journal for clinicians 65: 87-108, 2015.

3. Liu J, Sun Y, Zhang H, Ji D, Wu F, Tian H, Liu K, Zhang Y, Wu B and Zhang G: Theanine from tea and its semi-synthetic derivative $\mathrm{TBrC}$ suppress human cervical cancer growth and migration by inhibiting EGFR/Met-Akt/NF- $\kappa$ B signaling. Eur J Pharmacol 791: 297-307, 2016.
4. Munagala R, Aqil F, Jeyabalan J and Gupta RC: Tanshinone IIA inhibits viral oncogene expression leading to apoptosis and inhibition of cervical cancer. Cancer Lett 356: 536-546, 2015.

5. Kim B, Kim HS, Jung EJ, Lee JY, B KT, Lim JM and Song YS: Curcumin induces ER stress-mediated apoptosis through selective generation of reactive oxygen species in cervical cancer cells. Mol Carcinog 55: 918-928, 2016.

6. Promsong A, Chung WO, Satthakarn S and Nittayananta W: Ellagic acid modulates the expression of oral innate immune mediators: Potential role in mucosal protection. J Oral Pathol Med 44: 214-221, 2015.

7. Seo CS, Jeong SJ, Yoo SR, Lee NR and Shin HK: Quantitative analysis and in vitro anti-inflammatory effects of gallic acid, ellagic acid, and quercetin from radix sanguisorbae. Pharmacogn Mag 12: 104-108, 2016.

8. Baek B, Lee SH, Kim K, Lim HW and Lim CJ: Ellagic acid plays a protective role against UV-B-induced oxidative stress by up-regulating antioxidant components in human dermal fibroblasts. Korean J Physiol Pharmacol 20: 269-277, 2016.

9. Park SW, Kwon MJ, Yoo JY, Choi HJ and Ahn YJ: Antiviral activity and possible mode of action of ellagic acid identified in Lagerstroemia speciosa leaves toward human rhinoviruses. BMC Complement Altern Med 14: 171, 2014.

10. Zhao M, Tang S-N, Marsh JL, Shankar S and Srivastava RK: Ellagic acid inhibits human pancreatic cancer growth in Balb c nude mice. Cancer Lett 337: 210-217, 2013.

11. Ho CC, Huang AC, Yu CS, Lien JC, Wu SH, Huang YP, Huang HY, Kuo JH, Liao WY, Yang JS, et al: Ellagic acid induces apoptosis in TSGH8301 human bladder cancer cells through the endoplasmic reticulum stress- and mitochondria-dependent signaling pathways. Environ Toxicol 29: 1262-1274, 2014.

12. Hagiwara Y, Kasukabe T, Kaneko Y, Niitsu N and Okabe-Kado J: Ellagic acid, a natural polyphenolic compound, induces apoptosis and potentiates retinoic acid-induced differentiation of human leukemia HL-60 cells. Int J Hematol 92: 136-143, 2010.

13. Wang N, Wang ZY, Mo SL, Loo TY, Wang DM, Luo HB, Yang DP, Chen YL, Shen JG and Chen JP: Ellagic acid, a phenolic compound, exerts anti-angiogenesis effects via VEGFR-2 signaling pathway in breast cancer. Breast Cancer Res Treat 134: 943-955, 2012.

14. Narayanan BA, Geoffroy O, Willingham MC, Re GG and Nixon DW: p53/p21(WAF1/CIP1) expression and its possible role in $\mathrm{G} 1$ arrest and apoptosis in ellagic acid treated cancer cells. Cancer Lett 136: 215-221, 1999.

15. Eskandari E, Heidarian E, Amini SA and Saffari-Chaleshtori J: Evaluating the effects of ellagic acid on pSTAT3, pAKT, and pERK1/2 signaling pathways in prostate cancer PC 3 cells. J Cancer Res Ther, 2016.

16. Siveen KS, Sikka S, Surana R, Dai X, Zhang J, Kumar AP, Tan BK, Sethi G and Bishayee A: Targeting the STAT3 signaling pathway in cancer: Role of synthetic and natural inhibitors. Biochim Biophys Acta 1845: 136-154, 2014.

17. Yu H, Lee H, Herrmann A, Buettner R and Jove R: Revisiting STAT3 signalling in cancer: new and unexpected biological functions. Nat Rev Cancer 14: 736-746, 2014.

18. Shukla S, Shishodia G, Mahata S, Hedau S, Pandey A, Bhambhani S, Batra S, Basir SF, Das BC and Bharti AC: Aberrant expression and constitutive activation of STAT3 in cervical carcinogenesis: Implications in high-risk human papillomavirus infection. Mol Cancer 9: 282, 2010.

19. Chen CL, Hsieh FC, Lieblein J, Brown J, Chan C, Wallace J, Cheng G, Hall B and Lin J: Stat 3 activation in human endometrial and cervical cancers. Brit J Cancer 96: 591-599, 2007.

20. Ren C, Cheng X, Lu B and Yang G: Activation of interleukin-6/signal transducer and activator of transcription 3 by human papillomavirus early proteins 6 induces fibroblast senescence to promote cervical tumourigenesis through autocrine and paracrine pathways in tumour microenvironment. Eur J Cancer 49: 3889-3899, 2013.

21. Sobti R, Singh N, Hussain S, Suri V, Bharti A and Das B: Overexpression of STAT3 in HPV-mediated cervical cancer in a north Indian population. Mol Cell Biochem 330: 193-199, 2009.

22. Zellner A, Meixensberger J, Roggendorf W, Janka M, Hoehn H and Roosen K: DNA ploidy and cell-cycle analysis in intracranial meningiomas and hemangiopericytomas: a study with highresolution DNA flow cytometry. Int J Cancer 79: 116-20, 1998.

23. Engelke LH, Hamacher A, Proksch P and Kassack MU: Ellagic acid and resveratrol prevent the development of cisplatin resistance in the epithelial ovarian cancer cell line A2780. J Cancer 7: 353-363, 2016. 
24. Edderkaoui M, Odinokova I, Ohno I, Gukovsky I, Go VL, Pandol SJ and Gukovskaya AS: Ellagic acid induces apoptosis through inhibition of nuclear factor kappa B in pancreatic cancer cells. World J Gastroenterol 14: 3672-3680, 2008.

25. Salimi A, Roudkenar MH, Sadeghi L, Mohseni A, Seydi E, Pirahmadi N and Pourahmad J: Ellagic acid, a polyphenolic compound, selectively induces ROS-mediated apoptosis in cancerous B-lymphocytes of CLL patients by directly targeting mitochondria. Redox Biol 6: 461-471, 2015.

26. Kumar D, Basu S, Parija L, Rout D, Manna S, Dandapat J and Debata PR: Curcumin and Ellagic acid synergistically induce ROS generation, DNA damage, p53 accumulation and apoptosis in HeLa cervical carcinoma cells. Biomed Pharmacother 81: 31-37, 2016.

27. Chen HS, Bai MH, Zhang T, Li GD and Liu M: Ellagic acid induces cell cycle arrest and apoptosis through TGF- $\beta / \mathrm{Smad} 3$ signaling pathway in human breast cancer MCF-7 cells. Int J Oncol 46: 1730-1738, 2015.

28. Chung YC, Lu LC, Tsai MH, Chen YJ, Chen YY, Yao SP and Hsu CP: The inhibitory effect of ellagic Acid on cell growth of ovarian carcinoma cells. Evid Based Complement Alternat Med 2013: 306705, 2013.
29. Liu FT, Jia L, Wang P, Wang H, Farren TW and Agrawal SG: STAT3 and NF-kappaB cooperatively control in vitro spontaneous apoptosis and poor chemo-responsiveness in patients with chronic lymphocytic leukemia. Oncotarget 7: 32031-32045, 2016.

30. Li S, Priceman SJ, Xin H, Zhang W, Deng J, Liu Y, Huang J, Zhu W, Chen M, Hu W, et al: Icaritin inhibits JAK/STAT3 signaling and growth of renal cell carcinoma. PLoS One 8: e81657, 2013

31. Zhang $\mathrm{K}$ and Kumar R: Interferon-alpha inhibits cyclin E- and cyclin D1-dependent CDK-2 kinase activity associated with $R B$ protein and E2F in Daudi cells. Biochem Biophys Res Commun 200: 522-528, 1994.

This work is licensed under a Creative Commons Attribution-NonCommercial-NoDerivatives 4.0 International (CC BY-NC-ND 4.0) License. 